\title{
ASSISTÊNCIA DE ENFERMAGEM NO USO DA ELETROCIRURGIA: REVISÃO INTEGRATIVA
}

\author{
Nursing care in electrosurgery: integrative review
}

\section{El cuidado de enfermería en el uso de la electrocirugía: una revisión integradora}

Patrício de Almeida Costa ${ }^{1 *}$ (D), Eduarda Layane da Silva Buriti² (D), lara Mayanne de Castro Araújo² (D), Andrielly Cavalcante Fonseca ${ }^{2}$ (D), Adriana Montenegro de Albuquerque ${ }^{3}$ (D) Magaly Suênya de Almeida Pinto Abrante $^{4}$ (D)

RESUMO: Objetivo: Identificar o conhecimento científico produzido sobre os cuidados de enfermagem relacionados ao uso da eletrocirurgia no período intraoperatório. Método: Revisão integrativa, realizada nas bases de dados Literatura Latino-Americana e do Caribe em Ciências da Saúde (LILACS), PubMed, Cumulative Index to Nursing and Allied Health Literature (CINAHL) e na biblioteca virtual Scientific Electronic Library Online (SciELO), por meio dos descritores "eletrocirurgia", "cuidados de enfermagem" e "recuperação pós-cirúrgica melhorada", correlacionados pelo operador booleano and, nos idiomas português, inglês, espanhol e francês. Dos 213 estudos encontrados, sete compuseram a amostra. Utilizou-se o software Interface de R pour les Analyses Multidimensionnelles de Textes et de Questionnaires (IRAMUTEQ), por meio dos métodos de análise lexical e de similitude. Para a análise da qualidade metodológica, aplicou-se o nível de evidência científica, segundo recomendações da Agency for Healthcare Research and Quality (AHRQ). Resultados: Destacam-se publicações entre 2010 e 2012, com nível de evidência V, indexadas nas bases de dados LILACS e CINAHL. Verificaram-se, na nuvem de palavras, termos com maior frequência relativa: "paciente" ( $\mathrm{n}=14$ ); "risco cirúrgico" ( $\mathrm{n}=12$ ); "eletrocirurgia" ( $\mathrm{n}=10$ ); "enfermeiro" ( $\mathrm{n}=8$ ); "conhecimento" (n=6), "cuidado" (n=6) e "prevenção" (n=5). Feita a análise de similitude, identifica-se um leque semântico de palavras mais frequentes. Conclusão: Evidencia-se escassez de estudos, na literatura, com informações significativas para agregação de conhecimentos e construção de novos trabalhos.

Palavras-chave: Eletrocirurgia. Cuidados de enfermagem. Recuperação pós-cirúrgica melhorada.

ABSTRACT: Objective: To identify the scientific knowledge produced under the care of nursing related to the use of electrosurgery in the intraoperative period. Method: Integrative review based on the databases: Latin American and Caribbean Health Sciences Literature (LILACS), PubMed, Cumulative Index to Nursing and Allied Health Literature (CINAHL), and in the virtual library Scientific Electronic Library Online (SciELO), using the descriptors "electrosurgery", "nursing care" and "enhanced recovery after surgery", correlated by the Boolean operator and, in Portuguese, English, Spanish and French. Of the 213 studies we found, seven composed the sample. We used the Interface de R pour les Analyses Multidimensionnelles de Textes et de Questionnaires (IRAMUTEQ) software, through methods of lexical analysis and similitude. For the analysis of methodological quality, we applied the scientific level of evidence, according to recommendations from the Agency for Healthcare Research and Quality (AHRQ). Results: The publications of 2010 to 2012 stand out, with level of evidence V, indexed in LILACS and CINAHL. In the word cloud, we observed the following terms had higher relative frequency: "patient" $(\mathrm{n}=14)$; "surgical risk" ( $\mathrm{n}=12)$; "electrosurgery" ( $\mathrm{n}=10)$; "nurse" $(\mathrm{n}=8)$; "knowledge" ( $\mathrm{n}=6)$; "care" ( $\mathrm{n}=6)$; and "prevention" ( $\mathrm{n}=5)$. After the similitude analysis, we identified the semantic range of more frequent words. Conclusion: There are a few studies in the literature with significant information to aggregate knowledge and build new analyses.

Keywords: Electrosurgery. Nursing care. Enhanced recovery after surgery.

\footnotetext{
Acadêmico de Enfermagem do Centro de Educação e Saúde (CES), da Universidade Federal de Campina Grande (UFCG) - Cuité (PB), Brasil. ${ }^{2}$ Acadêmica de Enfermagem do CES/UFCG - Cuité (PB), Brasil.

${ }^{3}$ Doutora em Enfermagem pela Universidade Federal da Paraiba. Docente da UFCG - Cuité (PB), Brasil.

«Enfermeira, Doutoranda em Psicologia Clínica Intervenções em Psicanálise pela Universidade de São Paulo (USP). Docente da UFCG - Cuité (PB), Brasil.

*Autor correspondente: patricioalmeida13@hotmail.com

Recebido: 01/02/2021 - Aprovado: 16/05/2021

https://doi.org/10.5327/Z1414-4425202100020007
} 
RESUMEN: Objetivo: Identificar el conocimiento científico producido sobre la atención de enfermería relacionada con el uso de electrocirugía en el período intraoperatorio. Método: Revisión integradora, realizada en las bases de datos LILACS, PubMed, CINAHL y en la biblioteca virtual SciELO, a través de los descriptores Electrocirugía, Atención de enfermería y Recuperación posquirúrgica mejorada, correlacionados por el operador booleano and, en portugués, inglés, español y francés. Siete de los 213 estudios encontrados compusieron la muestra. Se utilizó el software IRAMUTEQ, mediante los métodos de análisis léxico y de similitud. Para el análisis de la calidad metodológica se aplicó el nivel de evidencia científica, según las recomendaciones de la Agency for Healthcare Research and Quality (AHRQ). Resultados: Destacan las publicaciones entre 2010 y 2012 , con nivel de evidencia V, indexadas en las bases de datos LILACS y CINAHL. En la nube de palabras se encontró términos con mayor frecuencia relativa: Paciente (n=14); Riesgo quirúrgico $(\mathrm{n}=12)$; Electrocirugía $(\mathrm{n}=10)$; Enfermero $(\mathrm{N}=08)$; Conocimiento $(\mathrm{N}=6)$, Atención $(\mathrm{N}=6)$ y Prevención ( $\mathrm{n}=5)$. Se identifica un rango semántico de palabras más frecuentes a partir del análisis de similitud. Conclusión: Se demostró la escasez de estudios en la literatura, con información significativa para la agregación del conocimiento y la construcción de nuevas obras.

Palabras clave: Electrocirugía. Atención de enfermería. Recuperación mejorada después de la cirugía.

\section{INTRODUÇÃo}

A eletrocirurgia, ou diatermia, consiste em um procedimento cirúrgico no qual se faz uso de correntes elétricas com polaridade de alta frequência em tecidos biológicos, com o manuseio de um material dielétrico para finalidades terapêuticas, como diérese e/ou hemostasia. Trata-se de uma prática antiga, que vem se aperfeiçoando ao longo do tempo com o avanço da tecnologia ${ }^{1}$.

Considera-se que a eletrocirurgia seja uma prática rotineira, amplamente utilizada nos centros cirúrgicos (CC), que possui diversos benefícios e garante maior qualidade na assistência perioperatória. Todavia, mesmo com o avanço das inovações tecnológicas, frequentemente são relatas ocorrências de complicações no ambiente cirúrgico, que apresentam risco potencial para o paciente, a equipe cirúrgica e toda a equipe multiprofissional ${ }^{2}$.

No tocante às principais complicações relacionadas ao uso da diatermia, destacam-se: inalação de fumaça, queimaduras, interferências no campo elétrico e em marcapassos. A queimadura é uma das complicações mais frequentes entre os pacientes submetidos ao procedimento ${ }^{3}$.

Dessa forma, a literatura aponta que a falta de comunicação entre os membros da equipe cirúrgica e o deficit de conhecimento sobre o uso dos equipamentos elétricos na cirurgia são fatores predisponentes ao aumento no risco de ocorrência de maiores complicações relacionadas ao procedimento. Ressalta-se, ainda, que o procedimento seguro se reflete na qualidade da assistência prestada ao paciente e realizada pela equipe cirúrgica nos períodos pré, intra e pós-operatório, objetivando diminuir possíveis danos, sequelas e eventos adversos ${ }^{2}$.
Na equipe cirúrgica, a enfermagem ocupa posição de destaque quanto à qualidade da assistência e à diminuição de riscos comuns no período perioperatório, com a prestação de cuidados sistematizados, que possibilitam o controle e o planejamento de estratégias que garantam ao paciente assistência integral, continuada, segura e humanizada, além de proporcionarem redução considerável de eventuais erros por toda a equipe. A possibilidade de uma aproximação direta ao paciente favorece a avaliação de suas condutas e instruções adequadas em todos os tempos cirúrgicos ${ }^{3,4}$.

Embora a importância e a amplitude da temática sejam reconhecidas internacionalmente, evidencia-se escassez na literatura de estudos direcionados para os cuidados de enfermagem no emprego da eletrocirurgia. O presente trabalho emergiu do conhecimento desenvolvido na disciplina “Tópicos Especiais: noções teóricas de enfermagem em centro cirúrgico e central de material e esterilização".

\section{OBJETIVO}

Identificar, por meio da literatura, o conhecimento científico produzido sobre os cuidados de enfermagem relacionados ao uso da eletrocirurgia no período intraoperatório.

\section{MÉTODO}

Revisão integrativa, cujo método permite reunir e analisar estudos científicos publicados na literatura de forma ampla e sistemática, compondo a síntese dos resultados 
sem modificar sua filiação epidemiológica, relacionando-os entre si, de forma crítica, para construção de um novo conhecimento5.

A pesquisa foi desenvolvida entre outubro e novembro de 2020 por quatro discentes do oitavo período do curso de Bacharelado em Enfermagem de uma instituição federal de ensino em expansão, orientadas por duas docentes. Foi conduzida durante a disciplina "Tópicos Especiais: Noções teóricas de enfermagem em centro cirúrgico e central de material de esterilização", ofertada no semestre letivo 2020.3, na modalidade remota em razão da pandemia do novo coronavírus.

Em relação às etapas da revisão integrativa, há similaridades com os diversos modelos apresentados na literatura científica. O modelo utilizado foi composto de seis etapas:

- definição da temática de interesse;

- formulação da pergunta norteadora;

- cruzamento dos descritores selecionados, correlacionados por meio do operador booleano "and" nas bases de dados selecionadas;

- seleção dos artigos relacionados com a temática e que atendiam os critérios de inclusão;

- recorte das informações extraídas ao longo das leituras dos artigos já existentes nas bases de dados;

- elaboração dos elementos textuais com base nas informações extraídas ${ }^{6}$.

Para a determinação da questão de pesquisa, utilizou-se a estratégia patient, intervention, comparison, outcomes (PICO). Trata-se de uma estratégia que permite a definição correta das evidências necessárias para a formulação da questão clínica de pesquisa, que potencializa a recuperação de evidências nas bases de dados, foca o alvo da pesquisa e evita a realização de buscas desnecessárias. Nessa estratégia, $\mathrm{P}=$ paciente ou problema, $\mathrm{I}=$ intervenção, $\mathrm{C}=$ comparação ou controle, $\mathrm{O}=$ outcomes ou desfecho ${ }^{7}$. Ressalta-se que o terceiro elemento, ou seja, a comparação, não foi utilizada no presente trabalho. O resultado foi a seguinte questão norteadora: quais são os cuidados de enfermagem relacionados ao uso da eletrocirurgia no período intraoperatório descritos na literatura científica?

Evidencia-se que a seleção e a busca dos estudos foi realizada de forma independente, a duplo cego, por dois autores do artigo a fim de evitar o viés de pesquisa e garantir a qualidade científica e a confiabilidade dos achados na literatura. Os resultados foram comparados e as diferenças solucionadas por consenso ou com a inclusão de um terceiro revisor, visando favorecer a consistência da seleção e a análise dos estudos. Foram consultadas as bases de dados Literatura Latino-Americana e do Caribe em Ciências da Saúde (LILACS), PubMed, Cumulative Index to Nursing and Allied Health Literature (CINAHL) e a biblioteca virtual Scientific Electronic Library Online (SciELO).

Utilizaram-se os descritores e suas combinações, correlacionadas pelo boleano $A N D$, nos idiomas português, inglês, espanhol e francês: "eletrocirurgia" " "electrosurgery"; "electrocirugia"; "électrochirurgie"), "cuidados de enfermagem" ("nursing care"; "atención de enfermería"; "soins infirmiers"), "enfermagem perioperatória" ("perioperative nursing"; "enfermería perioperatoria"; "soins infirmiers périopératoires"), "recuperação pós-cirúrgica melhorada” ("enhanced recovery after surgery"; "recuperación mejorada después de la cirugía"; without translation).

Ressaltam-se como critérios de inclusão: os textos disponíveis na íntegra; nos referidos idiomas; no formato on-line; e publicados nos últimos 11 anos (2009-2020). Foram excluídos artigos repetidos entre as bases de dados e literaturas consideradas cinzentas. Utilizou-se uma adaptação do modelo Preferred Reporting Items for Systematic Reviews and MetaAnalyses (PRISMA) (Figura 1) para a apresentação das etapas de seleção dos artigos.

Para a extração das informações da amostra final dos artigos, foi utilizado um instrumento com dados relacionados a título, autores, idioma, ano de publicação, método, nível de evidencia científica (NE), objetivos dos estudos e principais conclusões.

Visando ampliar ainda mais as possibilidades de análise, as considerações finais dos artigos selecionados foram empregadas como corpus textuais e submetidos ao processamento no software Interface de R pour les Analyses Multidimensionnelles de Textes et de Questionnaires (IRAMUTEQ). Esse programa organiza a distribuição do vocabulário de forma facilmente compreensível e visualmente clara ${ }^{8}$.

Na realização da análise textual pelo IRAMUTEQ, procedeu-se à separação do corpus em um único arquivo dividido em sete unidades de texto iniciais (UCI), sendo retidos 20 segmentos de texto, com aproveitamento de $60,34 \%$ do corpus. Posteriormente, os resultados foram apresentados considerando-se dois aspectos de análise: análise lexical — método nuvem de palavras - e análise de similitude.

Ademais, para a análise da qualidade metodológica, aplicou-se o modelo dos níveis de evidência científica da Agency for Healthcare Research and Quality (AHRQ), seguindo-se a seguinte classificação: 


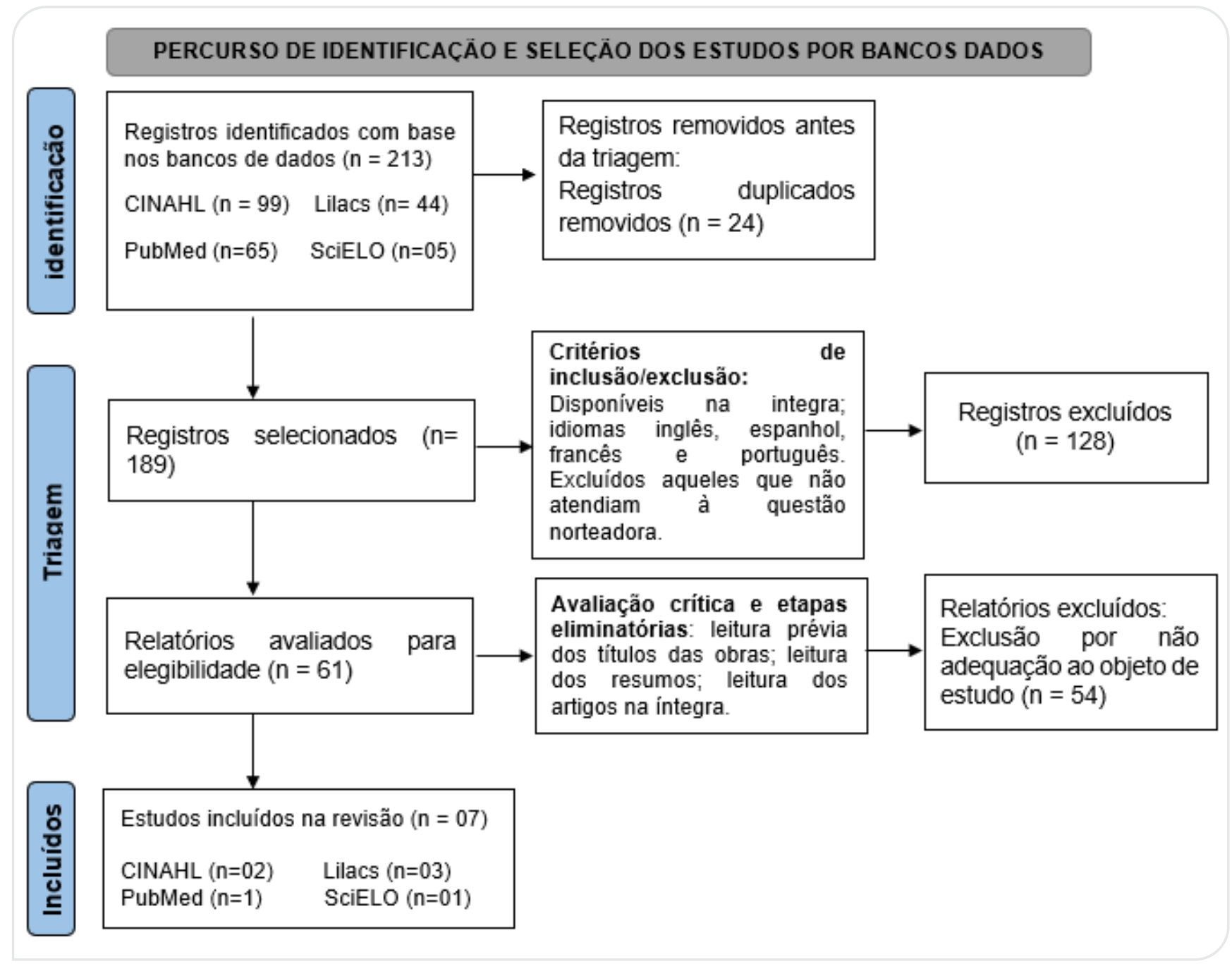

Figura 1. Fluxograma de identificação, triagem e seleção da produção científica, adaptado do Preferred Reporting Items for Systematic Reviews and Meta-Analyses.

- nível I: metanálise de múltiplos estudos controlados;

- nível II: estudo individual com delineamento experimental;

- nível III: estudo com delineamento quase-experimental como estudo sem randomização com grupo único pré e pós-teste, séries temporais ou caso-controle;

- nível IV: estudo com delineamento não experimental, como pesquisa descritiva correlacional e qualitativa ou estudos de caso;

- nível V: relatório de casos ou dado obtido de forma sistemática, de qualidade verificável ou dados de avaliação de programas;

- nível VI: opinião de autoridades respeitáveis, baseada na competência clínica ou na opinião de comitês de especialistas, incluindo interpretações de informações não baseadas em pesquisas? .

Os dados foram analisados, sintetizados e apresentados em forma de tabelas, nuvem de palavras e árvore de similitude. Para a construção do artigo de revisão integrativa, foram considerados e respeitados os aspectos éticos nas etapas supracitadas.

\section{RESULTADOS}

Sete artigos compuseram a amostra final do estudo, de um universo de 213, extraídos de periódicos diferentes e segundo 
critérios de elegibilidade. O Quadro 1 dispõe a caracterização bibliométrica dos artigos, composta de: número do artigo, autor/ano/idioma, título, método, base de dados e NE.

Identifica-se a predominância de publicações nos anos de 2010 e 2012, nível de evidência $V$, indexadas nas bases de dados LILACS e CINAHL. Há cinco artigos em português e dois em inglês. Em relação aos métodos das pesquisas, eles incluem: revisão integrativa, narrativa e estudos de delineamento descritivo e exploratório.

No Quadro 2, são sintetizados o objetivo, os principais resultados e a conclusão de cada artigo desta revisão integrativa.
Observa-se, no Quadro 2, que os verbos dos objetivos foram todos diferentes para descrever a temática, entre eles: buscar e avaliar (A1), elaborar (A2), analisar (A3), fornecer (A4), discutir (A5), caracterizar (A6) e identificar (A7). Nota-se, ainda, que em quatro dos artigos analisados (A1, A3, A4 e A7) a temática da assistência de enfermagem relacionada à eletrocirurgia se configura como o núcleo central da discussão dos estudos. Outros temas associados são: recomendações para a utilização da eletrocirurgia e a elaboração de protocolos de segurança (A2); complicações relacionadas ao procedimento (A5) e exposição da equipe à fumaça cirúrgica (A6).

Quadro 1. Categorização bibliométrica dos artigos incluídos na revisão integrativa.

\begin{tabular}{|c|c|c|c|c|c|}
\hline $\mathbf{N}$ & $\begin{array}{l}\text { Autor/Ano } \\
\text { Idioma }\end{array}$ & Título & Método & $\begin{array}{l}\text { Base de } \\
\text { Dados }\end{array}$ & NE \\
\hline A1 & $\begin{array}{c}\text { Brito e Galvão }{ }^{10} \\
2009 \\
\text { Português }\end{array}$ & $\begin{array}{l}\text { Os cuidados de enfermagem no uso da } \\
\text { eletrocirurgia }\end{array}$ & Revisão integrativa & LILACS & V \\
\hline A2 & $\begin{array}{c}\text { Brito e Galvão }{ }^{11} \\
2010 \\
\text { Português }\end{array}$ & $\begin{array}{l}\text { Protocolo de cuidados no uso de eletrocirurgia } \\
\text { monopolar }\end{array}$ & Revisão integrativa & LILACS & V \\
\hline A3 & $\begin{array}{c}\text { Olímpio et al. }{ }^{12} \\
2016 \\
\text { Português }\end{array}$ & $\begin{array}{l}\text { Electric scalpel usage and related safety } \\
\text { measures: integrative review }\end{array}$ & Revisão integrativa & CINAHL & V \\
\hline A4 & $\begin{array}{c}\text { Spruce e Braswell }{ }^{13} \\
2012 \\
\text { Inglês }\end{array}$ & $\begin{array}{l}\text { Implementing AORN recommended practices } \\
\text { for electrosurgery }\end{array}$ & Revisão narrativa & CINAHL & V \\
\hline A5 & $\begin{array}{c}\text { Afonso et al. }{ }^{14} \\
2010 \\
\text { Português }\end{array}$ & $\begin{array}{l}\text { Risco do uso do eletrocautério em pacientes } \\
\text { portadores de adornos metálicos }\end{array}$ & Revisão integrativa & SciELO & V \\
\hline A6 & $\begin{array}{l}\text { Steege et al. }{ }^{15} \\
2017 \\
\text { Inglês }\end{array}$ & $\begin{array}{l}\text { Secondhand smoke in the operating room? } \\
\text { Precautionary practices lacking for surgical smoke }\end{array}$ & $\begin{array}{l}\text { Descritivo- } \\
\text { exploratório }\end{array}$ & PubMed & IV \\
\hline A7 & $\begin{array}{l}\text { Parra et al. }{ }^{16} \\
2012 \\
\text { Português }\end{array}$ & $\begin{array}{c}\text { O conhecimento dos circulantes de sala sobre } \\
\text { a utilização do bisturi elétrico }\end{array}$ & $\begin{array}{l}\text { Descritivo- } \\
\text { exploratório }\end{array}$ & LILACS & IV \\
\hline
\end{tabular}

NE: nível de evidência; AORN: Association of periOperative Registered Nurses.

Quadro 2. Síntese de objetivo, principais resultados e conclusão das publicações.

\begin{tabular}{|c|c|c|c|}
\hline $\mathbf{N}$ & Objetivo & Principais Resultados & Conclusão \\
\hline$A 1^{10}$ & $\begin{array}{l}\text { Buscar e avaliar o } \\
\text { conhecimento científico } \\
\text { já produzido sobre os } \\
\text { cuidados de enfermagem } \\
\text { relacionados ao uso de } \\
\text { eletrocirurgia no período } \\
\text { intraoperatório. }\end{array}$ & $\begin{array}{l}\text { Foram apresentadas as seguintes categorias: } \\
\text { complicações decorrentes do uso da eletrocirurgia; } \\
\text { complicações decorrentes do uso da eletrocirurgia } \\
\text { de potência alta; complicações decorrentes do uso } \\
\text { da eletrocirurgia, outros equipamentos elétricos e } \\
\text { agentes antissépticos; conhecimento dos enfermeiros } \\
\text { sobre a unidade de eletrocirurgia; recomendações } \\
\text { práticas para o uso da eletrocirurgia. }\end{array}$ & $\begin{array}{c}\text { É imperativo para o enfermeiro } \\
\text { perioperatório o conhecimento técnico- } \\
\text { científico sobre o uso da eletrocirurgia } \\
\text { e as evidências encontradas fornecem } \\
\text { subsídios para a implantação de políticas e } \\
\text { procedimentos que garantam a segurança } \\
\text { do paciente, minimizando os riscos } \\
\text { potenciais dessa tecnologia. }\end{array}$ \\
\hline
\end{tabular}


Quadro 2. Continuação.

\begin{tabular}{|c|c|c|c|}
\hline $\mathbf{N}$ & Objetivo & Principais Resultados & Conclusão \\
\hline $\mathrm{A} 2^{11}$ & $\begin{array}{l}\text { Elaborar um protocolo } \\
\text { de cuidados para a } \\
\text { utilização da eletrocirurgia } \\
\text { monopolar. }\end{array}$ & $\begin{array}{l}\text { Para a elaboração do protocolo, sete artigos foram } \\
\text { analisados e as suas recomendações práticas foram } \\
\text { sintetizadas. O protocolo foi desenvolvido englobando } \\
\text { os seguintes itens: cuidados no pré-operatório, no } \\
\text { intraoperatório e no pós-operatório. }\end{array}$ & $\begin{array}{l}\text { A elaboração de protocolos de cuidados } \\
\text { possibilita a redução de erros e a } \\
\text { minimização de riscos, proporcionando } \\
\text { melhoria da qualidade da assistência } \\
\text { prestada, no pré-operatório, no } \\
\text { intraoperatório e no pós-operatório. }\end{array}$ \\
\hline$A 3^{12}$ & $\begin{array}{l}\text { Analisar evidências } \\
\text { científicas sobre a utilização } \\
\text { do bisturi elétrico e os } \\
\text { cuidados relacionados ao } \\
\text { uso desse equipamento. }\end{array}$ & $\begin{array}{l}\text { A estratégia de busca permitiu a análise de seis } \\
\text { artigos, que abordaram três temas principais: riscos } \\
\text { associados ao uso do bisturi elétrico, conhecimento } \\
\text { da equipe em relação ao uso desse instrumento } \\
\text { e papel do enfermeiro na prevenção de riscos } \\
\text { associados à eletrocirurgia. }\end{array}$ & $\begin{array}{c}\text { É necessário implementar ações } \\
\text { para que enfermeiros e técnicos de } \\
\text { enfermagem adquiram nível adequado de } \\
\text { conhecimentos e habilidades relacionadas } \\
\text { à segurança do paciente submetido à } \\
\text { eletrocirurgia. }\end{array}$ \\
\hline$A 4^{13}$ & $\begin{array}{c}\text { Fornecer orientação } \\
\text { aos enfermeiros } \\
\text { perioperatórios no uso e } \\
\text { cuidado de equipamentos } \\
\text { eletrocirúrgicos, incluindo } \\
\text { alta frequência, ultrassom } \\
\text { e modalidades de feixe de } \\
\text { argônio. }\end{array}$ & $\begin{array}{l}\text { As recomendações incluem selecionar unidades } \\
\text { eletrocirúrgicas e acessórios para compra, minimizar } \\
\text { o potencial de lesões em pacientes e membros da } \\
\text { equipe, definir precauções a ser tomadas durante a } \\
\text { cirurgia minimamente invasiva e formas de evitar } \\
\text { riscos de fumaça cirúrgica. }\end{array}$ & $\begin{array}{l}\text { As recomendações abordam educação, } \\
\text { competência, documentação, políticas, } \\
\text { procedimentos, qualidade garantia e } \\
\text { melhoria de desempenho. Enfermeiros } \\
\text { perioperatórios devem considerar o uso } \\
\text { de listas de verificação e cartazes de } \\
\text { segurança para lembrar os membros da } \\
\text { equipe dos perigos da eletrocirurgia e das } \\
\text { medidas a serem tomadas para minimizar } \\
\text { os riscos de lesões. }\end{array}$ \\
\hline$A 5^{14}$ & $\begin{array}{l}\text { Discutir os aspectos } \\
\text { relacionados às } \\
\text { complicações e ao emprego } \\
\text { adequado da eletrocirurgia. }\end{array}$ & $\begin{array}{l}\text { As lesões em eletrocirurgia monopolar ocorrem mais } \\
\text { frequentemente no local da placa dispersiva, mas } \\
\text { também em locais com eletrodos de monitoração e } \\
\text { em sítios de contato acidental com objetos de metal, } \\
\text { que funcionam como caminho alternativo para a } \\
\text { dispersão da eletricidade. As principais complicações } \\
\text { relacionadas ao uso da eletrocirurgia são as lesões } \\
\text { térmicas, seguidas por choques e interferência com } \\
\text { outros equipamentos e dispositivos elétricos. }\end{array}$ & $\begin{array}{l}\text { É essencial o conhecimento dos } \\
\text { fundamentos da eletrocirurgia, seu } \\
\text { uso correto, equipamento seguro, } \\
\text { monitoramento constante e investigação } \\
\text { imediata diante de quaisquer suspeitas } \\
\text { para minimizar o risco de acidentes em } \\
\text { pacientes com adornos metálicos. } \\
\text { A cooperação do paciente na obediência } \\
\text { das medidas preventivas de acidentes } \\
\text { deve ser obrigatória. }\end{array}$ \\
\hline$A 6^{15}$ & $\begin{array}{l}\text { Caracterizar o uso de } \\
\text { controles de exposição e } \\
\text { barreiras para a utilização } \\
\text { de ventilação de exaustão } \\
\text { local e equipamentos } \\
\text { de proteção individual } \\
\text { (incluindo proteção } \\
\text { respiratória) por } \\
\text { profissionais de saúde } \\
\text { que foram expostos } \\
\text { à fumaça cirúrgica } \\
\text { gerada por laser } \\
\text { ou procedimentos } \\
\text { eletrocirúrgicos. }\end{array}$ & $\begin{array}{l}\text { Ressalta-se que } 4.533 \text { entrevistados relataram } \\
\text { exposição à fumaça cirúrgica, } 4.500 \text { durante a } \\
\text { eletrocirurgia e } 1.392 \text { durante procedimentos de } \\
\text { cirurgia a laser. Foram entrevistados: enfermeiras } \\
\text { (56\%) e anestesiologistas ( } 21 \% \text { ). Apenas } 14 \% \text { dos } \\
\text { expostos durante a eletrocirurgia relataram que o } \\
\text { exaustor de ventilação local sempre foi usado durante } \\
\text { esses procedimentos, enquanto } 47 \% \text { relataram o } \\
\text { uso durante a cirurgia a laser. Poucos entrevistados } \\
\text { relataram o uso de proteção respiratória. }\end{array}$ & $\begin{array}{l}\text { Os resultados do estudo podem ser usados } \\
\text { para aumentar a conscientização sobre o } \\
\text { uso marginal de controles de exposição e } \\
\text { impedimentos para seu uso. }\end{array}$ \\
\hline$A 7^{16}$ & $\begin{array}{l}\text { Identificar o conhecimento } \\
\text { dos profissionais de } \\
\text { enfermagem na sala } \\
\text { cirúrgica quanto à utilização } \\
\text { do bisturi elétrico. }\end{array}$ & $\begin{array}{l}\text { Embora o uso do bisturi elétrico seja frequente no } \\
\text { centro cirúrgico, só houve treinamento eficaz a } 54 \% \\
\text { dos usuários. Quanto aos cuidados específicos com os } \\
\text { pacientes portadores de marcapasso e que precisam } \\
\text { utilizar o bisturi elétrico, } 72 \% \text { dos entrevistados } \\
\text { desconheciam tais cuidados. }\end{array}$ & $\begin{array}{c}\text { Há falhas no treinamento da equipe } \\
\text { de enfermagem de centro cirúrgico no } \\
\text { emprego do bisturi elétrico. Sugere-se } \\
\text { que seja viabilizada educação permanente } \\
\text { sobre esse assunto, de modo a garantir a } \\
\text { qualidade no atendimento e a segurança } \\
\text { do paciente cirúrgico. }\end{array}$ \\
\hline
\end{tabular}


Na construção da nuvem de palavras, os termos que obtiveram maior frequência relativa foram: "paciente" ( $\mathrm{n}=14)$; "risco cirúrgico" ( $\mathrm{n}=12)$; "eletrocirurgia" $(\mathrm{n}=10)$; "enfermeiro" $(\mathrm{n}=8)$; "conhecimento" ( $n=6)$, "cuidado" ( $n=6)$ e "prevenção" $(n=5)$, que tomaram posição central e tiveram maior tamanho em relação às outras palavras, conforme representado na Figura 2.

$\mathrm{Na}$ análise de relação entre os termos selecionados no processamento dos dados com base na análise de similitude (Figura 3), observa-se um leque semântico de palavras mais frequentes: "paciente", "eletrocirurgia", "enfermeiro" e "risco cirúrgico".

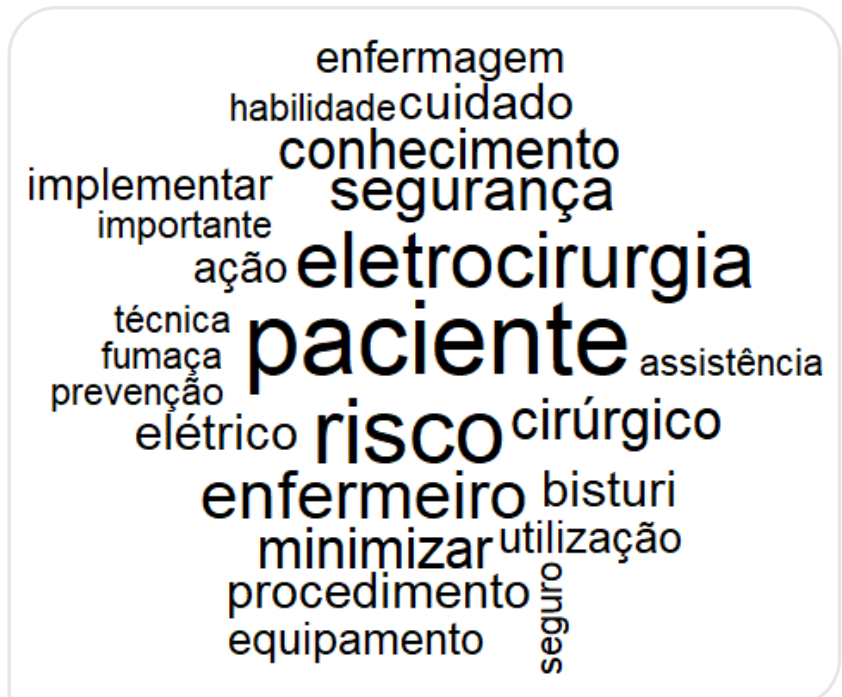

Figura 2. Nuvem de palavras.

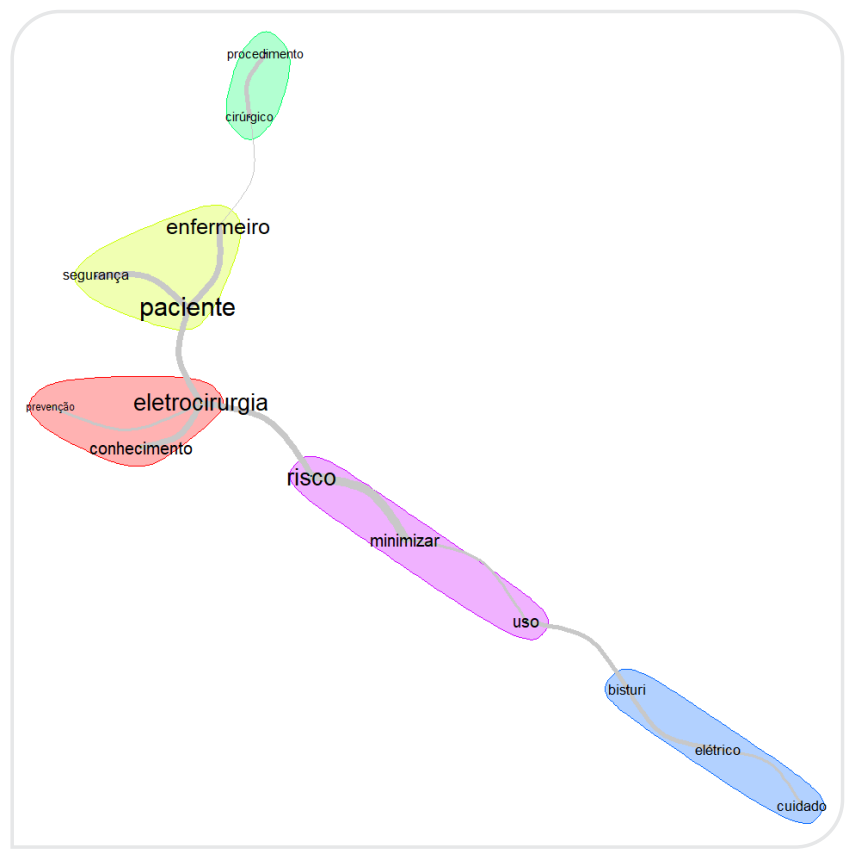

Figura 3. Análise de similitude dos corpus textuais.

\section{DISCUSSÃO}

$\mathrm{Na}$ análise dos artigos, os estudos apontaram os principais riscos e as complicações que podem ocorrer pelo uso inadequado do eletrocautério. Destacam-se como fatores de risco: tempo de exposição à corrente elétrica, uso do sistema monopolar, falta de comunicação entre os membros da equipe cirúrgica, danificação ou uso inadequado na rede de gazes, deficit de conhecimento dos profissionais a respeito do funcionamento, da utilização e dos cuidados necessários ao manuseio seguro do bisturielétrico ${ }^{13,14,16}$.

Identifica-se que o paciente está exposto a riscos diretos ou indiretos relacionados ao procedimento cirúrgico, como citado anteriormente. O tempo de exposição à corrente elétrica ou duração da corrente é um fator que influencia nos efeitos da sua passagem pelo corpo humano, de modo que, quanto maior o tempo de exposição, maiores os efeitos e os riscos de lesão ${ }^{12,17}$.

As principais complicações identificadas na sumarização dos artigos selecionados foram as queimaduras, os choques elétricos e a inalação da fumaça cirúrgica, que levam a um comprometimento significativo do paciente. Ressalta-se, em uma pesquisa, que o risco de lesão está associado ao surgimento de queimaduras resultantes de três causas secundárias: trauma térmico não intencional ou uso inapropriado do eletrodo ativo; trauma térmico indesejável na região da placa; e a terceira causa pode ocorrer quando a corrente elétrica assume um caminho indesejável através do corpo do paciente e não do eletrodo de dispersão ${ }^{14}$.

Salienta-se que os choques são complicações da unidade eletrocirúrgica, comumente confundidos com queimaduras, que ocorrem quando o cirurgião segura o instrumento no tecido a ser cauterizado. A fim de evitar o choque do eletrodo ativo, ele deve ser colocado na região de interesse antes da ativação. Materiais e adornos metálicos podem potencializar o risco, aumentando, em proporção, a ocorrência de lesões ${ }^{1,12,14}$.

Pesquisadores observaram que a fumaça dispersada na eletrocirurgia pode conter gazes e vapores tóxicos, como benzeno, cianeto de hidrogênio, formaldeído, bioaerossóis, material celular vivo e morto e vírus. Uma das recomendações para conter esse dano é o uso de sistemas de evacuação de fumaça para que, assim, sejam reduzidos os potenciais riscos agudos e crônicos à saúde tanto dos pacientes como da equipe cirúrgica, ${ }^{1,17,18}$.

Faz-se necessário destacar que o bloco cirúrgico é um ambiente terapêutico que visa promover segurança e bem-estar ao paciente. Cabe ao enfermeiro reconhecer e minimizar 
os possíveis riscos para evitar complicações, planejando e traçando intervenções de enfermagem que contribuam para a concretização do bem-estar e a reabilitação do paciente. Para o atendimento ao paciente em tratamento cirúrgico, é imprescindível uma assistência de enfermagem perioperatória que vise planejar e implementar ações e que seja categorizada por um processo dinâmico e sistemático ${ }^{14,19}$.

No tocante à análise da árvore de similitudes, foi possível considerar, por meio de conexões dos vocábulos, que o sentido dado ao cuidado de enfermagem no período intraoperatório da eletrocirurgia visa garantir a segurança do paciente durante o procedimento no intuito de diminuir riscos e complicações associadas, para o que algumas recomendações e cuidados devem ser seguidos.

Nesse sentido, a equipe de enfermagem é fundamental no desempenho dos cuidados profissionais baseados em evidências científicas que supram as necessidades do paciente. Ademais, os procedimentos de enfermagem realizados na eletrocirurgia são subdivididos em cuidados com o paciente e com o ambiente, durante o perioperatório. Destacam-se os cuidados de certificação de dados pessoais, procedimento cirúrgico correto, indicação, exames laboratoriais, sinais vitais nos parâmetros normais, instruções e preparação do paciente no pré-operatório ${ }^{20}$.

Além dos cuidados no transporte e na recepção do paciente no CC, destacam-se a utilização de solução antisséptica aquosa em vez de alcoólica, a adoção da posição cirúrgica do paciente e o uso racional do oxigênio. Ademais, os pacientes que são usuários de marca-passo devem ser constantemente monitorados, pois, apesar da modernidade dos dispositivos, eles ainda estão sujeitos à interferência da corrente elétrica. Outros cuidados, como monitorização dos parâmetros do paciente durante o procedimento, posicionamento adequado da placa de dispersão, acompanhamento na sala de recuperação pós-anestésica, monitoração e curativos cirúrgicos devem ser rigorosamente seguidos ${ }^{11,19,21}$.

A eletrocirurgia é uma prática rotineira nos CC. Dessa forma, cuidados de enfermagem no ambiente perioperatório são essenciais, como: preparo da $\mathrm{SO}$; ajuste dos focos e da potência do bisturi elétrico, utilizando-se valores que não produzam faíscas; colocação dos campos cirúrgicos o mais longe possível de fontes de calor; certificação do funcionamento de todas as tomadas e aparelhos da SO; providenciar o material que será utilizado; atentar para que não ocorra acúmulo de líquidos inflamáveis em qualquer cavidade do corpo do paciente; o paciente não deve entrar em contato com objetos de metal; cabos e eletrodos devem ser verificados antes do uso a fim de garantir que todo o isolamento esteja intacto, entre outras condutas ${ }^{10,12}$.

$\mathrm{Na}$ eletrocirurgia, os cuidados de enfermagem perpassam os cuidados com o paciente propriamente dito, sendo necessário atenção para a manutenção dos equipamentos a serem utilizados e todo o local onde será realizado o procedimento cirúrgico ${ }^{11,22}$.

Quanto às limitações deste estudo, destaca-se o baixo nível de evidência científica entre a amostra dos artigos e a escassez na literatura de estudos sobre a temática. Evidencia-se, dessa forma, a necessidade da construção de novas produções científicas que venham a agregar e preencher as lacunas presentes na literatura atual, contribuindo com informações significativas para integrar novos conhecimentos e favorecer a assistência de enfermagem qualificada ao paciente quando submetido à eletrocirurgia.

Todavia, apesar das limitações, os achados do estudo podem ser utilizados em âmbito acadêmico e profissional no intuito de favorecer a aquisição de conhecimentos atualizados a respeito dos cuidados de enfermagem relacionados ao uso da eletrocirurgia no período intraoperatório, além de promover, indiretamente, a assistência qualificada e segura ao paciente cirúrgico.

\section{CONCLUSÃO}

Este estudo possibilitou identificar evidências científicas dos cuidados necessários em relação ao uso da eletrocirurgia e seus riscos associados. Adotaram-se publicações que ressaltam a importância do diálogo efetivo entre os membros da equipe multiprofissional para o desenvolvimento de ações de prevenção que minimizem os riscos dos procedimentos relacionados à eletrocirurgia, incluindo o monitoramento constante do equipamento seguro. Além disso, destacou-se a necessidade da autocapacitação, por meio de estratégias de educação continuada e permanente entre os profissionais da equipe.

Ressalta-se a necessidade da execução de estratégias que possibilitem aos profissionais de enfermagem adquirir conhecimento técnico-científico adequado e competências relacionadas à segurança do paciente submetido à eletrocirurgia. A meta é diminuir a possibilidade de ocorrência de eventos adversos durante o procedimento, destacando-se entre eles as queimaduras, os choques e a inalação da fumaça cirúrgica, que interferem diretamente na integridade física dos pacientes e dos profissionais. 


\section{REFERÊNCIAS}

1. Bisinotto FMB, Dezena RA, Martins LB, Galvão MC, Martins Sobrinho J, Calçado MS. Burns related to electrosurgery: report of two cases. Braz J Anesthesiol [Internet]. 2017 [acessado em $18 \mathrm{dez}$. 2020];67(5):527-34. Disponível em: https://www.scielo.br/pdf/rba/ v67n5/pt_0034-7094-rba-67-05-0527.pdf. https://doi.org/10.1016/j. bjan.2016.03.003

2. Farias IP, Almeida TG, Lopes RF, Torres BA, Silva RRSM, Trindade RFC, et al. The safe use of eletrosurgery. Rev Enferm UFPE Online [Internet]. 2019 [acessado em $17 \mathrm{dez}$. 2020];13(2):538-41. Disponível em: https://periodicos.ufpe.br/revistas/revistaenfermagem/article/ view/237989/31379

3. Gutierres L, Guedes dos Santos J, Cechinel Peiter C, Antunes Menegon F, Sebold L, Lorenzini Erdmann A. Good practices for patient safety in the operating room: nurses' recommendations. Rev Bras Enferm [Internet]. 2018 [acessado em 11 nov. 2020];71(Supl. 6):2775-82. Disponivel em: https://www.scielo.br/pdf/reben/v71s6/0034-7167reben-71-s6-2775.pdf. https://doi.org/10.1590/0034-7167-2018-0449

4. Macedo JKSS, Vasconcelos EL. 0 uso seguro da eletrocirurgia no período intraoperatório: evidências para o cuidado de enfermagem. Res Soc Dev [Internet]. 2021 [acessado em 21 dez. 2020];10(1):e1210111203. Disponível em: https://rsdjournal.org/ index.php/rsd/article/view/11203/10222. https://doi.org/10.33448/ rsd-v10i1.11203

5. Souza LMM, Vieira CM, Serverino S, Antunes V. A metodologia de revisão integrativa da literatura em enfermagem. Rev Investig Enferm [Internet]. 2017 [acessado em 17 nov. 2020];21(2):17-26. Disponível em: https://www.researchgate.net/publication/321319742_Metodologia_ de_Revisao_Integrativa_da_Literatura_em_Enfermagem

6. Mendes KDS, Silveira RC de CP, Galvão CM. Revisão integrativa: método de pesquisa para a incorporação de evidências na saúde e na enfermagem. Texto Context - Enferm. 2008;17(4):758-64. https:// doi.org/10.1590/S0104-07072008000400018

7. Santos CMC, Pimenta CAM, Nobre MRC. The pico strategy for the research question construction and evidence search. Rev Latino-Am Enferm [Internet]. 2007 [acessado em 17 out. 2020];15(3):508-11. Disponível em: https://www.scielo.br/pdf/rlae/v15n3/v15n3a23.pdf. https://doi.org/10.1590/S0104-11692007000300023

8. Acauan LV, Abrantes CV, Stipp MAC, Trotte LAC, Paes GO, Queiroz ABA. Use of the Iramuteq ${ }^{\circledR}$ Software for quantitative data analysis in nursing: a reflective essay. Rev Min Enferm [Internet]. 2020 [acessado em 9 out. 2020];24:e-1326. Disponível em: https:// cdn.publisher.gn1.link/reme.org.br/pdf/en_e1326.pdf. https://doi. org/10.5935/1415-2762.20200063

9. Stetler CB, Morsi D, Rucki S, Broughton S, Corrigan B, Fitsgerald $\mathrm{J}$, et al. Utilization-focused integrative reviews in a nursing service. Appl Nurs Res [Internet]. 1998 [acessado em $1{ }^{\circ}$ maio 2021];11(4):195206. Disponível em: https://www.sciencedirect.com/science/article/ pii/S0897189798803297

10. Brito MFP, Galvão CM. Os cuidados de enfermagem no uso da eletrocirurgia. Rev Gaúcha Enferm [Internet]. 2009 [acessado em 11 dez. 2020];30(2):319-27. Disponível em: https://seer.ufrgs.br/index. php/RevistaGauchadeEnfermagem/article/view/5733/6691
11. Brito MFP, Galvão CM. Protocolo de cuidados no uso de eletrocirurgia monopolar. Rev SOBECC [Internet]. 2010 [acessado em $10 \mathrm{dez}$. 2020];15(3):33-8. Disponível em: https://revista.sobecc.org.br/sobecc/ issue/viewlssue/19/pdf_48

12. Olímpio MAC, Sousa VEC, Ponte MAV. Electric scalpel usage and related safety measures: integrative review. Rev SOBECC [Internet]. 2016 [acessado em 10 dez. 2020];21(3):154-61. Disponível em: https:// revista.sobecc.org.br/sobecc/article/view/33/pdf_1

13. Spruce L, Braswell ML. Implementing AORN Recommended Practices for Electrosurgery. AORN J [Internet]. 2012 [acessado em 13 dez. 2020];95(3):373-87. Disponível em: https://doi.org/10.1016/j. aorn.2011.12.018

14. Afonso CT, Silva AL, Fabrini DS, Afonso CT, Côrtes MGW, Sant'Anna LL. Risco do uso do eletrocautério em pacientes portadores de adornos metálicos. ABCD Arq Bras Cir Dig [Internet]. 2010 [acessado em 12 nov. 2020;23(3):183-6. Disponível em: https://www. scielo.br/pdf/abcd/v23n3/v23n3a10.pdf. https://doi.org/10.1590/ S0102-67202010000300010

15. Steege AL, Boiano JM, Sweeney MH. Secondhand smoke in the operating room? Precautionary practices lacking for surgical smoke. Am J Ind Med [Internet]. 2016 [acessado em 22 nov. 2020];59(1 1):102031. Disponivel em: http://doi.wiley.com/10.1002/ajim.22614. https:// doi.org/10.1002/ajim.22614

16. Parra RLC, Giannasttasio MB, Diniz TRZ. O conhecimento dos circulantes de sala sobre a utilização do bisturi elétrico. Rev SOBECC [Internet]. 2012 [acessado em 12 nov. 2020];17(4):24-32. Disponível em: https://revista.sobecc.org.br/sobecc/article/view/170/pdf-a

17. Ball K. Surgical smoke evacuation guidelines: compliance among perioperative nurses. AORN J [Internet]. 2010 [acessado em 30 nov. 2020];92(2):e1-e23. Disponível em: http://dx.doi.org/10.1016/j. aorn.2009.10.026

18. Croke L. Guideline for electrosurgical safety. AORN J [Internet]. 2020 [acessado em 22 nov. 2020];112(1):P9-P11. Disponivel em: https:// doi.org/10.1002/aorn.13124

19. Souza IB, Tenório HAA, Gomes Junior EL, Sarmento Neto ML, Almeida BR, Marques ES. Percepção do cliente no perioperatório sobre o cuidado de enfermagem no centro cirúrgico. Rev Eletrônica Acervo Saúde [Internet]. 2019 [acessado em 29 nov. 2020];(26):e840. Disponível em: https://doi.org/10.25248/reas.e840.2019

20. Jost MT, Viegas K, Caregnato RCA. Systematization of perioperatory nursing assistance in patient safety: an integrative review. Rev SOBECC [Internet]. 2018 [acessado em 25 nov. 2020];23(4):218-25. Disponível em: https://revista.sobecc.org.br/sobecc/article/view/440/pdf

21. Pignot $G$. Role of the nurse in the implementation of enhanced recovery after surgery. Prog en Urol [Internet]. 2019 [acessado em $1^{\circ}$ dez. 2020];29(15):904-7. Disponivel em: https://doi.org/10.1016/j. purol.2019.08.269

22. O'Riley M. Electrosurgery in perioperative practice. J Perioper Pract [Internet]. 2010 [acessdao em $1^{\circ}$ dez. 2020];20(9):329-33. Disponível em: https://doi.org/10.1177/175045891002000903 